\title{
Common Fixed Point Theorems of New Contractive Conditions in Fuzzy Metric Spaces
}

\author{
Jiang Zhu, ${ }^{1}$ Yuan Wang, ${ }^{1}$ and Shin Min Kang ${ }^{2}$ \\ ${ }^{1}$ School of Mathematics and Statistics, Jiangsu Normal University, Xuzhou 221116, China \\ ${ }^{2}$ Department of Mathematics and RINS, Gyeongsang National University, Jinju 660-701, Republic of Korea
}

Correspondence should be addressed to Shin Min Kang; smkang@gnu.ac.kr

Received 26 February 2013; Accepted 8 May 2013

Academic Editor: Tamaki Tanaka

Copyright (c) 2013 Jiang Zhu et al. This is an open access article distributed under the Creative Commons Attribution License, which permits unrestricted use, distribution, and reproduction in any medium, provided the original work is properly cited.

\begin{abstract}
Some new limit contractive conditions in fuzzy metric spaces are introduced, by using property (E.A), some common fixed point theorems for four maps are proved in GV-fuzzy metric spaces. As an application of our results, some new contractive conditions are presented, and some common fixed point theorems are proved under these contractive conditions. The contractive conditions presented in this paper contain or generalize many contractive conditions that appeared in the literatures. Some examples are given to illustrate that our results are real generalizations for the results in the references and to show that our limit contractive conditions are important for the existence of fixed point.
\end{abstract}

\section{Introduction}

The theory of fuzzy sets was first introduced by Zadeh [1], after many authors introduced the notion of fuzzy metric spaces in different ways (see [2-5]). In particular, Kramosil and Michálek [4] generalized the concept of probabilistic metric space given by Menger [6] to the fuzzy framework. Later on, George and Veeramani [2] modified the concept of fuzzy metric space introduced by Kramosil and Michálek and defined the Hausdorff and first countable topology on the modified fuzzy metric space. Actually, this topology can also be constructed on each fuzzy metric space in the sense of Kramosil and Michálek and it is metrizbale [2,7]. Other recent contributions to the study of fuzzy metric spaces in the sense of [2] may be found in $[8,9]$. Since then, many authors have proved fixed point and common fixed point theorems in fuzzy metric spaces in the sense of [2]. Especially, we want to emphasize that some common fixed point theorems for $\varphi$-type contraction maps in fuzzy metric spaces have been recently obtained in [10-16].

Quite recently, Miheț [13] proved some existence theorems of common fixed point for two self-mappings $f, g$ of a fuzzy metric space $(X, M, *)$ under the following contractive condition:

$$
\begin{gathered}
M(f x, f y, t) \\
\geq \varphi(\min \{M(g x, g y, t), M(f x, g x, t), M(f y, g y, t), \\
M(f x, g y, t), M(f y, g x, t)\})
\end{gathered}
$$

for all $x, y \in X$ and $t>0$, where $\varphi:[0,1] \rightarrow[0,1]$ is continuous and nondecreasing on $[0,1]$, and $\varphi(x)>x$ for all $x \in[0,1]$.

C. Vetro and P. Vetro [15] proved some existence theorems of common fixed point for two self-mappings $f, g$ of a fuzzy metric space $(X, M, *)$ under the following contractive condition:

$$
\begin{aligned}
& \frac{1}{M(f x, f y, t)}-1 \\
& \leq r\left(\frac{1}{\min \{M(g x, g y, t), M(f x, g x, t), M(f y, g y, t)\}}-1\right)
\end{aligned}
$$


for all $x, y \in X$ and $t>0$, where $r:[0,+\infty) \rightarrow[0,+\infty)$ with $r(\tau)<\tau$ for every $\tau>0$, an upper semicontinuous function.

Gopal et al. [10] proved some existence theorems of common fixed point for four self-mappings $A, B, S$ and $T$ of a fuzzy metric space $(X, M, *)$ under the following contractive condition:

$$
\begin{aligned}
& \frac{1}{M(A x, B y, t)}-1 \\
& \leq r\left(\frac{1}{\min \{M(S x, T y, t), M(A x, S x, t), M(B y, T y, t)\}}-1\right)
\end{aligned}
$$

for all $x, y \in X$ and $t>0$, where $r:[0,+\infty) \rightarrow[0,+\infty)$ with $r(\tau)<\tau$ for every $\tau>0$, an upper semicontinuous function.

Imdad and Ali [11], Vijayaraju and Sajath [16] proved some existence theorems of common fixed point for four selfmappings $A, B, S$, and $T$ of a fuzzy metric space $(X, M, *)$ under the following contractive condition:

$$
\begin{aligned}
& M(A x, B y, t) \\
& \quad \geq \phi(\min \{M(S x, T y, t), M(S x, A x, t), M(B y, T y, t)\})
\end{aligned}
$$

for all $x, y \in X$ and $t>0$, where $\phi:[0,1] \rightarrow[0,1]$ with $\phi(s)>s$ whenever $0<s<1$ is a continuous or increasing and left-continuous function.

Imdad et al. [12] proved some existence theorems of common fixed point for four self-mappings $f, g, S$, and $T$ of a fuzzy metric space $(X, M, *)$ under the following contractive condition:

$$
\begin{aligned}
& M(f x, g y, t) \\
& \geq \varphi(\min \{M(S x, T y, t), M(f x, S x, t), \\
&M(f y, T y, t), M(f x, T y, t), M(f y, S x, t)\})
\end{aligned}
$$

for all $x, y \in X$ and $t>0$, where $\varphi:[0,1] \rightarrow[0,1]$ is continuous and nondecreasing on $[0,1]$, and $\varphi(x)>x$ for all $x \in[0,1]$.

Shen et al. [14] proved an existence theorem of fixed point for self-mapping $T$ of a fuzzy metric space $(X, M, *)$ under the following contractive condition:

$$
\varphi(M(T x, T y, t)) \leq k(t) \cdot \varphi(M(x, y, t))
$$

for all $x, y \in X$ and $t>0$, where $\varphi:[0,1] \rightarrow[0,1]$ satisfies the following properties:

(P1) $\varphi$ is strictly decreasing and left continuous;

(P2) $\varphi(\lambda)=0$ if and only if $\lambda=1$.

Furthermore, let $k$ be a function from $(0, \infty)$ into $(0,1)$.

The purpose of this paper is to present limit contractive conditions to unify all of these $\varphi$-type nonlinear contractive conditions. Then, by using property (E.A), some common fixed point theorems for four maps are proved in GV-fuzzy metric spaces. As an application of our limit contraction condition, we present some new $\varphi$-type integral contractive conditions and some common fixed point theorems for four maps in GV-fuzzy metric spaces under these contractive conditions. Our results generalize the corresponding results in [10-16]. Some examples are given to illustrate that our results are real generalizations for the results in the references and show that our limit contractive conditions are important for the existence of fixed point.

For the reader's convenience, we recall some terminologies from the theory of fuzzy metric spaces, which will be used in what follows.

Definition 1 (see [4]). A continuous $t$-norm in the sense of Kramosil and Michálek is a binary operation * on $[0,1]$ satisfying the following conditions:

(1) $*$ is associative and commutative,

(2) $a * 1=a$ for all $a \in[0,1]$,

(3) $a * b \leq c * d$ whenever $a \leq c$ and $b \leq d$ for all $a, b, c, d \in$ $[0,1]$,

(4) the mapping $*:[0,1] \times[0,1] \rightarrow[0,1]$ is continuous.

Three typical examples of continuous $t$-norm are $a * b=$ $\max \{a+b-1,0\}, a * b=a b$, and $a * b=\min \{a, b\}$.

Definition 2 (see [2]). A fuzzy metric space in the sense of George and Veeramani is a triple $(X, M, *)$, where $X$ is a nonempty set, $M$ is a fuzzy set on $X^{2} \times(0, \infty)$, and $*$ is a continuous $t$-norm such that the following conditions are satisfied for all $x, y, z \in X$ and $t, s>0$

$(\mathrm{GV}-1) M(x, y, t)>0$;

$(\mathrm{GV}-2) M(x, y, t)=1$ if and only if $x=y$;

$(\mathrm{GV}-3) M(x, y, t)=M(y, x, t)$;

$(\mathrm{GV}-4) M(x, y, t) * M(y, z, s) \leq M(x, z, t+s)$;

$(\mathrm{GV}-5) M(x, y, \cdot):(0, \infty) \rightarrow[0,1]$ is continuous.

In what follows, fuzzy metric spaces in the sense of George and Veeramani will be called $G V$-fuzzy metric spaces.

Lemma 3 (see [17]). Let $(X, M, *)$ be a $G V$-fuzzy metric space. Then $M(x, y, t)$ is non-decreasing with respect to $t$ for all $x, y \in$ X.

Definition 4 (see [13]). Let $(X, M, *)$ be a GV-fuzzy metric space. Then one has the following:

(1) a sequence $\left\{x_{n}\right\}$ in $X$ is said to be convergent to $x \in X$ if $\lim _{n \rightarrow \infty} M\left(x_{n}, x, t\right)=1$ for all $t>0$.

(2) a sequence $\left\{x_{n}\right\}$ in $X$ is said to be Cauchy sequence if $\lim _{n \rightarrow \infty} M\left(x_{n}, x_{n+p}, t\right)=1$ for all $t>0$ and $p \in \mathbb{N}$.

(3) a fuzzy metric space is called complete if every Cauchy sequence converges in $X$.

Lemma 5 (see [5]). Let $(X, M, *)$ be a $G V$-fuzzy metric space. Then $M$ is a continuous function on $X^{2} \times(0, \infty)$. 
Definition 6 (see [18]). Let $(X, M, *)$ be a GV-fuzzy metric space. Then two self-mappings $A$ and $S$ of $(X, M, *)$ satisfy property (E.A) if there exists a sequence $\left\{x_{n}\right\}$ in $X$ and $z$ in $X$ such that $\left\{A x_{n}\right\}$ and $\left\{S x_{n}\right\}$ converge to $z$ that is, for any $t>0$,

$$
\lim _{n \rightarrow \infty} M\left(A x_{n}, z, t\right)=\lim _{n \rightarrow \infty} M\left(S x_{n}, z, t\right)=1 .
$$

Definition 7 (see [18]). Let $(X, M, *)$ be a GV-fuzzy metric space. Then two pairs of self-mappings $A, S$ and $B, T$ of $(X, M, *)$ are said to share common property (E.A) if there exist sequences $\left\{x_{n}\right\}$ and $\left\{y_{n}\right\}$ in $X$ such that, for any $t>0$,

$$
\begin{aligned}
\lim _{n \rightarrow \infty} M\left(A x_{n}, z, t\right) \\
\quad=\lim _{n \rightarrow \infty} M\left(S x_{n}, z, t\right) \\
\quad=\lim _{n \rightarrow \infty} M\left(B y_{n}, z, t\right)=\lim _{n \rightarrow \infty} M\left(T y_{n}, z, t\right)=1
\end{aligned}
$$

for some $z \in X$.

Definition 8 (see [19]). Let $(X, M, *)$ be a GV-fuzzy metric space. Then two self-mappings $A$ and $S$ of $(X, M, *)$ are said to be weak compatible if they commute at their coincidence point; that is,

$$
A x=S x \text { implies } S A x=A S x .
$$

\section{Main Results}

Let $(X, M, *)$ be a GV-fuzzy metric space, and let $A, B, S$, and $T$ be self-mappings of $(X, M, *)$. For any $x, y \in X$ and $t>0$, we define

$$
\begin{gathered}
\operatorname{Min}(x, y, t) \\
=\min \{M(S x, T y, t), M(S x, A x, t), M(T y, B y, t), \\
M(S x, B y, t), M(T y, A x, t)\} .
\end{gathered}
$$

Consider that $(C 1) 0<\underline{\lim }_{n \rightarrow \infty} \operatorname{Min}\left(x_{n}, y_{n}, t\right)=L(t)<$ 1 implies that $\varlimsup_{n \rightarrow \infty} M\left(A x_{n}, B y_{n}, t\right)>L(t)$ for all $t>0$ and any sequence $\left\{x_{n}\right\}$ and $\left\{y_{n}\right\}$ in $X$.

Theorem 9. Let $(X, M, *)$ be a $G V$-fuzzy metric space, and $A$, $B, S$, and $T$ be self-mappings of $(X, M, *)$ such that one has the following:

(1) (C1) holds;

(2) A, S are weakly compatible and B, T are weakly compatible;

(3) A, S satisfy property (E.A) or B, T satisfy property (E.A);

(4) $A X \subset T X$ and $B X \subset S X$;

(5) one of the range of the mappings $A, B, S$, or $T$ is a closed subspace of $X$.

Then $A, B, S$, and $T$ have a unique common fixed point.
Proof. Suppose that $B, T$ satisfy the property (E.A). Then there exists a sequence $\left\{x_{n}\right\}$ in $X$ such that

$$
\lim _{n \rightarrow \infty} M\left(B x_{n}, z, t\right)=\lim _{n \rightarrow \infty} M\left(T x_{n}, z, t\right)=1
$$

for all $t>0$ and some $z \in X$.

Since $B X \subset S X$, there exists a sequence $\left\{y_{n}\right\}$ in $X$ such that $B x_{n}=S y_{n}$. Hence $\lim _{n \rightarrow \infty} M\left(S y_{n}, z, t\right)=1$ for all $t>0$.

Suppose that $S X$ is a closed subspace of $X$. Then $z=S u$ for some $u \in X$. Subsequently, we have that

$$
\begin{aligned}
\lim _{n \rightarrow \infty} M\left(B x_{n}, S u, t\right) \\
=\lim _{n \rightarrow \infty} M\left(T x_{n}, S u, t\right) \\
=\lim _{n \rightarrow \infty} M\left(S y_{n}, S u, t\right)=1
\end{aligned}
$$

for all $t>0$. Then by using Lemma 5 we have that

$$
\begin{gathered}
\lim _{n \rightarrow \infty} M\left(T x_{n}, B x_{n}, t\right)=1, \\
\lim _{n \rightarrow \infty} M\left(T x_{n}, A u, t\right)=M(S u, A u, t) .
\end{gathered}
$$

Thus, we have that

$$
\begin{gathered}
\varliminf_{n \rightarrow \infty} \operatorname{Min}\left(u, x_{n}, t\right) \\
=\varliminf_{n \rightarrow \infty} \min \left\{M\left(S u, T x_{n}, t\right), M(S u, A u, t),\right. \\
M\left(T x_{n}, B x_{n}, t\right), M\left(S u, B x_{n}, t\right), \\
\left.M\left(T x_{n}, A u, t\right)\right\} \\
=M(S u, A u, t)=\lim _{n \rightarrow \infty} M\left(B x_{n}, A u, t\right)
\end{gathered}
$$

for any $t>0$; that is,

$$
\varliminf_{n \rightarrow \infty} \operatorname{Min}\left(u, x_{n}, t\right)=\varlimsup_{n \rightarrow \infty} M\left(A u, B x_{n}, t\right)=M(S u, A u, t) .
$$

If $S u \neq A u$, then there exists $t>0$ such that $0<$ $M(S u, A u, t)<1$. This and $(C 1)$ imply that

$$
\varlimsup_{n \rightarrow \infty} M\left(A u, B x_{n}, t\right)>\varliminf_{n \rightarrow \infty} \operatorname{Min}\left(u, x_{n}, t\right) .
$$

This is a contradiction. Thus, we have that $S u=A u$. The weak compatibility of $A$ and $S$ implies that $A S u=S A u$, and then $A A u=A S u=S A u=S S u$.

On the other hand, since $A X \subset T X$, there exists $v \in X$ such that $A u=T v$. Since for any $t>0$

$$
\begin{aligned}
& \operatorname{Min}(u, v, t) \\
& =\min \{M(S u, T v, t), M(S u, A u, t), M(T v, B v, t), \\
& M(S u, B v, t), M(T v, A u, t)\}=M(A u, B v, t),
\end{aligned}
$$

by $(C 1)$, we get $M(A u, B v, t)=1$, which implies that $A u=$ $B v$; that is, $A u=S u=T v=B v$. The weak compatibility of $B$ and $T$ implies that $B T v=T B v$ and $T T v=T B v=B T v=B B v$. 
Let us show that $A u$ is a common fixed point of $A, B, T$, and $S$. Since

$$
\begin{aligned}
& \operatorname{Min}(A u, v, t) \\
&= \min \{M(S A u, T v, t), M(S A u, A A u, t), \\
&M(T v, B v, t), M(S A u, B v, t), M(T v, A A u, t)\} \\
&=\min \{M(A A u, B v, t), 1,1, M(A A u, B v, t), \\
&M(T v, A A u, t)\} \\
&=M(A A u, B v, t),
\end{aligned}
$$

by $(C 1)$, we get that $M(A A u, B v, t)=1$, which implies that $A A u=B v$. Therefore, $A A u=S A u=B v=A u$, and $A u$ is a common fixed point of $A$ and $S$. Similarly, we can prove that $B v$ is a common fixed point of $B$ and T. Noting that $A u=B v$, we conclude that $A u$ is a common fixed point $A, B, T$, and $S$. The proof is similar when $T X$ is assumed to be a closed subspace of $X$. The cases in which $A X$ or $B X$ is a closed subspace of $X$ are similar to the cases in which $T X$ or $S X$, respectively, is closed since $A X \subset T X$ and $B X \subset S X$. If $A u=B u=T u=S u=u$ and $A v=B v=T v=S u=v$, then

$$
\begin{gathered}
\operatorname{Min}(u, v, t) \\
=\min \{M(S u, T v, t), M(S u, A u, t), M(T v, B v, t), \\
M(S u, B v, t), M(T v, A u, t)\} \\
=\min \{M(A u, T v, t), 1,1, M(S u, B v, t), \\
M(T v, A u, t)\} \\
=M(A u, B v, t) .
\end{gathered}
$$

Therefore, by $(C 1)$, we have $u=v$; that is, the common fixed point is unique. This completes the proof.

To introduce some integral contractive conditions, let $h$ : $[0,1] \rightarrow \mathbb{R}_{+}$be nonnegative, Lebesgue integrable, and satisfy

$$
\int_{0}^{\varepsilon} h(t) d t>0
$$

for each $0<\varepsilon \leq 1$. We denote $d=\int_{0}^{1} h(t) d t$.

Theorem 10. Let $(X, M, *)$ be a $G V$-fuzzy metric space, and $A$, $B, S$, and $T$ be self-mappings of $(X, M, *)$. If one of the following conditions is satisfied

$(C 2)$ There exists a function $\omega:[0, d] \rightarrow[0, d]$ such that for any $0<s<d, \omega(s)<s, \varlimsup_{u \rightarrow s} \omega(u)<s$ and for any $x, y \in X, t \in(0, \infty), 0<\operatorname{Min}(x, y, t)<1$ implies that

$$
\omega\left(\int_{0}^{M(A x, B y, t)} h(s) d s\right) \geq \int_{0}^{\operatorname{Min}(x, y, t)} h(s) d s
$$

(C3) There exists a function $\phi:[0, d] \rightarrow[0, d]$ such that, for any $0<s<d, \phi(s)>s, \underline{\lim }_{t \rightarrow s} \phi(t)>s$, and for any $x, y \in X, t \in(0, \infty), 0<\operatorname{Min}(x, y, t)<1$ implies that

$$
\int_{0}^{M(A x, B y, t)} h(s) d s \geq \phi\left(n \int_{0}^{\operatorname{Min}(x, y, t)} h(s) d s\right) .
$$

Then (C1) holds.

Proof. $(C 2) \Rightarrow(C 1)$. Assume that (C2) holds. If $\left\{x_{n}\right\}$ and $\left\{y_{n}\right\}$ in $X$ and $0<\underline{\lim }_{n \rightarrow \infty} \operatorname{Min}\left(x_{n}, y_{n}, t\right)=L(t)<1$, then

$$
\begin{aligned}
\varlimsup_{n \rightarrow \infty} \omega & \left(\int_{0}^{M\left(A x_{n}, B y_{n}, t\right)} h(\tau) d \tau\right) \\
& \geq \varlimsup_{n \rightarrow \infty} \int_{0}^{\operatorname{Min}\left(x_{n}, y_{n}, t\right)} h(\tau) d \tau \\
& \geq \int_{0}^{\lim _{n \rightarrow \infty} \operatorname{Min}\left(x_{n}, y_{n}, t\right)} h(\tau) d \tau=\int_{0}^{L(t)} h(\tau) d \tau .
\end{aligned}
$$

If $\overline{\lim }_{n \rightarrow \infty} M\left(A x_{n}, B y_{n}, t\right)=1$, then $\overline{\lim }_{n \rightarrow \infty} M\left(A x_{n}, B y_{n}, t\right)$ $>L(t)$. If $\varlimsup_{n \rightarrow \infty} M\left(A x_{n}, B y_{n}, t\right)=r(t)<1$, then there exists a subsequence $\left\{M\left(A x_{n_{i}}, B y_{n_{i}}, t\right)\right\}$ such that

$$
\lim _{i \rightarrow \infty} M\left(A x_{n_{i}}, B y_{n_{i}}, t\right)=\varlimsup_{n \rightarrow \infty} M\left(A x_{n}, B y_{n}, t\right)=r(t) .
$$

Thus,

$$
\begin{aligned}
\varlimsup_{n \rightarrow \infty} & \int_{0}^{M\left(A x_{n}, B y_{n}, t\right)} h(\tau) d \tau \\
& =\lim _{i \rightarrow \infty} \int_{0}^{M\left(A x_{n_{i}}, B y_{n_{i}}, t\right)} h(\tau) d \tau \\
& =\int_{0}^{r(t)} h(\tau) d \tau>\varlimsup_{u \rightarrow \int_{0}^{r(t)} h(\tau) d \tau} \omega(u) \\
& \geq \varlimsup_{i \rightarrow \infty} \omega\left(\int_{0}^{\operatorname{Min}\left(x_{n_{i}}, y_{n_{i}}, t\right)} h(\tau) d \tau\right) \geq \int_{0}^{L(t)} h(\tau) d \tau .
\end{aligned}
$$

This implies that

$$
\varlimsup_{n \rightarrow \infty} M\left(A x_{n}, B y_{n}, t\right)=r(t)>L(t) .
$$

Thus, (C1) holds.

$(C 3) \Rightarrow(C 1)$. Assume that (C3) holds. If $\left\{x_{n}\right\}$ and $\left\{y_{n}\right\}$ in $X$ and $0<\underline{\lim }_{n \rightarrow \infty} \operatorname{Min}\left(x_{n}, y_{n}, t\right)=L(t)<1$, then there exists a subsequence $\left\{\operatorname{Min}\left(x_{n_{i}}, y_{n_{i}}, t\right)\right\}$ such that $\lim _{i \rightarrow \infty} \operatorname{Min}\left(x_{n_{i}}, y_{n_{i}}, t\right)=L(t)$. This implies that

$$
\begin{gathered}
\varliminf_{i \rightarrow \infty} \phi\left(\int_{0}^{\operatorname{Min}\left(x_{n_{i}}, y_{n_{i}}, t\right)} h(\tau) d \tau\right) \\
\geq \underline{\lim }_{t \rightarrow \int_{0}^{L(t)} h(\tau) d \tau} \phi(t)>\int_{0}^{L(t)} h(\tau) d \tau \\
\quad=\lim _{i \rightarrow \infty} \int_{0}^{\operatorname{Min}\left(x_{n_{i}}, y_{n_{i}}, t\right)} h(\tau) d \tau .
\end{gathered}
$$


It follows from

$$
\int_{0}^{M(A x, B y, t)} h(\tau) d \tau \geq \phi\left(\int_{0}^{\operatorname{Min}(x, y, t)} h(\tau) d \tau\right)
$$

that we can get

$$
\begin{aligned}
\varlimsup_{n \rightarrow \infty} & \int_{0}^{M\left(A x_{n}, B y_{n}, t\right)} h(\tau) d \tau \\
& =\int_{0}^{\varlimsup_{n \rightarrow \infty} M\left(A x_{n}, B y_{n}, t\right)} h(\tau) d \tau \\
& \geq \varlimsup_{n \rightarrow \infty} \phi\left(\int_{0}^{\operatorname{Min}\left(x_{n}, y_{n}, t\right)} h(\tau) d \tau\right) \\
& \geq \varlimsup_{i \rightarrow \infty} \phi\left(\int_{0}^{\operatorname{Min}\left(x_{n_{i}}, y_{n_{i}}, t\right)} h(\tau) d \tau\right) \\
& \geq \varliminf_{i \rightarrow \infty} \phi\left(\int_{0}^{\operatorname{Min}\left(x_{n_{i}}, y_{n_{i}}, t\right)} h(\tau) d \tau\right)>\int_{0}^{L(t)} h(\tau) d \tau .
\end{aligned}
$$

This implies that $\varlimsup_{n \rightarrow \infty} M\left(A x_{n}, B y_{n}, t\right)>L(t)$; that is, $(C 1)$ holds.

It follows from Theorems 9 and 10 that we have the following fixed point theorems for integral type contractive mappings.

Theorem 11. Let $(X, M, *)$ be a $G V$-fuzzy metric space, and let $A, B, S$, and $T$ be self-mappings of $(X, M, *)$ such that one has the following

(1) one of (C2)-(C3) holds;

(2) A, S are weakly compatible and B, T are weakly compatible;

(3) A, S satisfy the property (E.A) or B, T satisfy the property (E.A);

(4) $A X \subset T X$ and $B X \subset S X$;

(5) one of the range of the mappings $A, B, S$, or $T$ is a closed subspace of $X$.

Then $A, B, S$, and $T$ have a unique common fixed point.

In (C2) and (C3), by taking $h(t) \equiv 1$, we have the following contractive conditions.

(C4) There exists a function $\omega:[0,1] \rightarrow[0,1]$ such that for any $0<s<1, \omega(s)<s, \overline{\lim }_{u \rightarrow s} \omega(u)<s$ and for any $x, y \in X, t \in(0, \infty), 0<\operatorname{Min}(x, y, t)<1$ implies that

$$
\omega(M(x, y, t)) \geq \operatorname{Min}(x, y, t) .
$$

$(C 5)$ There exists a function $\phi:[0,1] \rightarrow[0,1]$ such that, for any $0<s<1, \phi(s)>s, \underline{\lim }_{t \rightarrow s} \phi(t)>s$, and for any $x, y \in X, t \in(0, \infty), 0<\operatorname{Min}(x, y, t)<1$ implies that

$$
M(A x, B y, t) \geq \phi(\operatorname{Min}(x, y, t)) .
$$

Corollary 12. Let $(X, M, *)$ be a $G V$-fuzzy metric space, and let $A, B, S$, and $T$ be self-mappings of $(X, M, *)$ such that one has the following:

(1) one of (C4)-(C5) holds;

(2) A, S are weakly compatible and B, T are weakly compatible;

(3) A, S satisfy property (E.A) or B, T satisfy the property (E.A);

(4) $A X \subset T X$ and $B X \subset S X$;

(5) one of the range of the mappings $A, B, S$, or $T$ is a closed subspace of $X$.

Then $A, B, S$ and $T$ have a unique common fixed point.

Remark 13. As a special case of (C4), we can take function $\omega:[0,1] \rightarrow[0,1]$ as one of the following:

(1) $\omega$ is nonincreasing, for any $0<s<1, \omega(s)<s$, $\overline{\lim }_{u \rightarrow s^{-}} \omega(u)<s ;$

(2) $\omega$ is an upper semicontinuous function such that, for any $0<s<1, \omega(s)<s$;

(3) $\omega$ is non-increasing and left-upper semicontinuous such that $\omega(s)<s$ for any $0<s<1$.

As a special case of (C5), we can take function $\phi$ : $[0,1] \rightarrow[0,1]$ as one of the follows:

(1) $\phi$ is a nondecreasing and left-continuous function such that, for any $0<s<1, \phi(s)>s$;

(2) $\phi$ is a a lower semi-continuous function such that for any $0<s<1, \phi(s)>s$;

(3) $\phi(s)=s+\psi(s)$, where $\psi:[0,1] \rightarrow[0,1]$ is a continuous function with for any $0<s<1, \psi(s)>0$.

From Corollary 12, we have the following corollaries.

Corollary 14. Let $(X, M, *)$ be a $G V$-fuzzy metric space, and let $A, B, S$, and $T$ be self-mappings of $(X, M, *)$ such that one has the following:

(1) there exists an upper semicontinuous function $r$ : $[0,+\infty) \rightarrow[0,+\infty)$ with $r(s)<s$ for any $s>0$ such that for all $x, y \in X$ and $t>0$

$$
\frac{1}{M(A x, B y, t)}-1 \leq r\left(\frac{1}{\operatorname{Min}(x, y, t)}-1\right)
$$

(2) A, S are weakly compatible and B, T are weakly compatible;

(3) A, S satisfy property (E.A) or B, T satisfy property (E.A);

(4) $A X \subset T X$ and $B X \subset S X$;

(5) one of the range of the mappings $A, B, S$ or $T$ is a closed subspace of $X$.

Then $A, B, S$, and $T$ have a unique common fixed point. 
Proof. Define function $\phi:[0,1] \rightarrow[0,1]$ by

$$
\phi(t)= \begin{cases}0 & \text { if } t=0 \\ \frac{1}{1+r((1 / t)-1)} & \text { if } t \in(0,1] .\end{cases}
$$

Then, for any $s>0$,

$$
\begin{aligned}
\varliminf_{t \rightarrow s} \phi(t) & =\frac{1}{1+\overline{\lim }_{t \rightarrow s} r((1 / t)-1)} \\
& =\frac{1}{1+r((1 / s)-1)}>\frac{1}{1+(1 / s)-1}=s,
\end{aligned}
$$

and (32) can be rewritten as: for any $x, y \in X, t \in(0, \infty)$, $0<\operatorname{Min}(x, y, t)<1$ implies that

$$
M(A x, B y, t) \geq \phi(\operatorname{Min}(x, y, t)) .
$$

That is, (C5) holds. Then the conclusion can be deduced from Corollary 12. This completes the proof.

Corollary 15. Let $(X, M, *)$ be a $G V$-fuzzy metric space, and $A, B, S$, and $T$ be self-mappings of $(X, M, *)$ such that one has the following:

(1) there exists a strictly decreasing and left continuous function $\varphi:[0,1] \rightarrow[0,1]$ with $\varphi(\lambda)=0$ if and only if $\lambda=1$ and function $k:(0, \infty) \rightarrow(0,1)$ such that for all $x, y \in X(x \neq y)$ and $t>0$

$\varphi(M(A x, B y, t)) \leq k(t) \cdot \varphi(\operatorname{Min}(x, y, t)) ;$

(2) A, S are weakly compatible and B, T are weakly compatible;

(3) A, S satisfy property (E.A) or B, T satisfy property (E.A);

(4) $A X \subset T X$ and $B X \subset S X$;

(5) one of the range of the mappings $A, B, S$, or $T$ is a closed subspace of $X$.

Then, $A, B, S$ and $T$ have a unique common fixed point.

Proof. Define function $\phi:[0,1] \times(0,+\infty) \rightarrow[0,1]$ by

$$
\phi(s, t)=\varphi^{-1}(k(t) \varphi(s)) .
$$

Since $\varphi$ is strictly decreasing and left continuous, we have that $\varphi^{-1}$ is strictly decreasing and right continuous and $\phi(s, t)$ is increasing in $s$. Then we have that

$$
\varliminf_{u \rightarrow s^{-}} \phi(u, t)=\varphi^{-1}(k(t) \varphi(s)) .
$$

Also we have that $\phi(u, t) \geq \phi(s, t)$ for $u>s$; this shows that

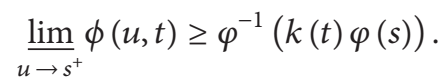

That is, we can get that

$$
\varliminf_{u \rightarrow s} \phi(u, t) \geq \phi(s, t)=\varphi^{-1}(k(t) \varphi(s))>\varphi^{-1}(\varphi(s))=s,
$$

and (36) can be rewritten as follows: for any $x, y \in X, t \in$ $(0, \infty), 0<\operatorname{Min}(x, y, t)<1$ implies that

$$
M(A x, B y, t) \geq \phi(\operatorname{Min}(x, y, t), t) .
$$

If $\left\{x_{n}\right\}$ and $\left\{y_{n}\right\}$ in $X$ and $0<\underline{\lim }_{n \rightarrow \infty} \operatorname{Min}\left(x_{n}, y_{n}, t\right)=L(t)<$ 1 , then there exists a subsequence $\left\{\operatorname{Min}\left(x_{n_{i}}, y_{n_{i}}, t\right)\right\}$ such that $\lim _{i \rightarrow \infty} \operatorname{Min}\left(x_{n_{i}}, y_{n_{i}}, t\right)=L(t)$. This shows that

$$
\begin{aligned}
& \varliminf_{i \rightarrow \infty} \phi\left(\operatorname{Min}\left(x_{n_{i}}, y_{n_{i}}, t\right), t\right)
\end{aligned}
$$

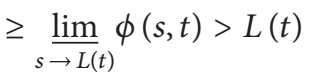

$$
\begin{aligned}
& =\lim _{i \rightarrow \infty} \operatorname{Min}\left(x_{n_{i}}, y_{n_{i}}, t\right) \text {. }
\end{aligned}
$$

It follows from $M(A x, B y, t) \geq \phi(\operatorname{Min}(x, y, t), t)$ that we can get

$$
\begin{aligned}
& \varlimsup_{n \rightarrow \infty} M\left(A x_{n}, B y_{n}, t\right) \\
& \geq \varlimsup_{n \rightarrow \infty} \phi\left(\operatorname{Min}\left(x_{n}, y_{n}, t\right), t\right) \\
& \geq \varlimsup_{i \rightarrow \infty} \phi\left(\operatorname{Min}\left(x_{n_{i}}, y_{n_{i}}, t\right), t\right) \\
& \geq \lim _{i \rightarrow \infty} \phi\left(\operatorname{Min}\left(x_{n_{i}}, y_{n_{i}}, t\right), t\right)>L(t) .
\end{aligned}
$$

Thus, we have that $(C 1)$ holds. The conclusion can be deduced from Theorem 9. This completes the proof.

Remark 16. The main result Theorems 3.1 and 2.1 in [13] are the special cases of our Theorem 9 and Corollary 14 for $A=B=f$ and $S=T=g$. Especially, it follows from Corollary 12 that the condition " $\varphi$ is nondecreasing" is not needed. Therefore, our results improve and generalize the results in [13].

Let $(X, M, *)$ be a GV-fuzzy metric space, and let $A, B, S$, and $T$ be self-mappings of $(X, M, *)$. For any $x, y \in X$ and $t>0$, we define

$$
\begin{aligned}
\widetilde{\operatorname{Min}} & (x, y, t) \\
& =\min \{M(S x, T y, t), M(S x, A x, t), M(T y, B y, t)\} .
\end{aligned}
$$

Consider that $(\widetilde{C} 1) 0<\underline{\lim }_{n \rightarrow \infty} \widetilde{\operatorname{Min}}\left(x_{n}, y_{n}, t\right)=\widetilde{L}(t)<1$ implies $\varlimsup_{n \rightarrow \infty} M\left(A x_{n}, B y_{n}, t\right)>\widetilde{L}(t)$ for all $t>0$ and any sequence $\left\{x_{n}\right\}$ and $\left\{y_{n}\right\}$ in $X$.

By $(44)$, we can write the conditions $(\widetilde{C} 2)-(\widetilde{C} 5)$ which correspond to the conditions $(C 2)-(C 5)$ in Theorem 9, Theorem 10 and Corollary 12 by replacing $\operatorname{Min}(x, y, t)$ with $\widetilde{\operatorname{Min}}(x, y, t)$. It is similar to the proof of Theorem 10, we can know that one of $(\widetilde{C} 4)-(\widetilde{C} 5)$ can imply $(\widetilde{C} 1)$ holds. 
Corollary 17. Let $(X, M, *)$ be a GV-fuzzy metric space, and $A, B, S$ and $T$ be self-mappings of $(X, M, *)$ such that one has the following:

(1) $(\widetilde{C} 1)$ or one of $(\widetilde{C} 4)-(\widetilde{C} 5)$ holds;

(2) $A, S$ are weakly compatible and $B, T$ are weakly compatible;

(3) A, S satisfy property (E.A) or B, T satisfy property (E.A);

(4) $A X \subset T X$ and $B X \subset S X$;

(5) one of the range of the mappings $A, B, S$, or $T$ is a closed subspace of $X$.

Then $A, B, S$, and $T$ have a unique common fixed point.

Proof. It is clear that we only need to prove Corollary 17 for $(\widetilde{C} 1)$. Assume that sequence $\left\{x_{n}\right\}$ and $\left\{y_{n}\right\}$ in $X, 0<$ $\underline{\lim }_{n \rightarrow \infty} \operatorname{Min}\left(x_{n}, y_{n}, t\right)=L(t)<1$. Then

$$
\lim _{n \rightarrow \infty} \widetilde{\operatorname{Min}}\left(x_{n}, y_{n}, t\right)=\widetilde{L}(t) \geq \lim _{n \rightarrow \infty} \operatorname{Min}\left(x_{n}, y_{n}, t\right)=L(t) .
$$

If $\underline{\lim }_{n \rightarrow \infty} \overparen{\operatorname{Min}}\left(x_{n}, y_{n}, t\right)=\widetilde{L}(t)<1$, then by $(\widetilde{C} 1)$ we have that

$$
\varlimsup_{n \rightarrow \infty} M\left(A x_{n}, B y_{n}, t\right)>\widetilde{L}(t) \geq L(t) .
$$

If $\underline{\lim }_{n \rightarrow \infty} \overparen{\operatorname{Min}}\left(x_{n}, y_{n}, t\right)=\widetilde{L}(t)=1$, then, for any $n$, either $\widetilde{\operatorname{Min}}\left(x_{n}, y_{n}, t\right)<1$ or $\overparen{\operatorname{Min}}\left(x_{n}, y_{n}, t\right)=1$. If $\overparen{\operatorname{Min}}\left(x_{n}, y_{n}, t\right)<1$, then by $(\widetilde{C} 1)$ we have that $M\left(A x_{n}, B y_{n}, t\right)>\widetilde{\operatorname{Min}}\left(x_{n}, y_{n}, t\right)$. If $\widehat{\operatorname{Min}}\left(x_{n}, y_{n}, t\right)=1$, then by (44) we have that $A x_{n}=B y_{n}$. By $(\mathrm{GV}-2)$ we get that $M\left(A x_{n}, B y_{n}, t\right)=1=\overparen{\operatorname{Min}}\left(x_{n}, y_{n}, t\right)$. Thus, $(\widetilde{C} 1)$ implies that $M\left(A x_{n}, B y_{n}, t\right) \geq \widetilde{\operatorname{Min}}\left(x_{n}, y_{n}, t\right)$ for any $n$. This implies that

$$
\begin{aligned}
\varlimsup_{n \rightarrow \infty} M & \left(A x_{n}, B y_{n}, t\right) \\
& \geq \varliminf_{n \rightarrow \infty} \widetilde{\operatorname{Min}}\left(x_{n}, y_{n}, t\right)=\widetilde{L}(t)=1>L(t) .
\end{aligned}
$$

Therefore, $(\widetilde{C} 1)$ implies that $(C 1)$ holds. Then by Theorem 9 we know that $A, B, S$, and $T$ have a unique common fixed point. This completes the proof.

Remark 18. In Theorem 9 and Corollaries 12-17, conditions (3), (4), and (5) can be replaced by the following conditions:

$\left(3^{\prime}\right) A, S$ and $B, T$ share the common property (E.A);

$\left(4^{\prime}\right)$ the range, of the mappings $S$ and $T$ are closed subspaces of $X$.

The proof can be got by properly modifying the proof of Theorem 9, Corollaries 12-17. Thus, from Theorem 9, Corollaries 12-17 and Remark 13, we can see that our results generalize and improve the results in [10-16].

Example 19. Let $X=[0,1], M(x, y, t)=t /(t+|x-y|)$ for every $x, y \in X$ and $t>0$ and $a * b=\min \{a, b\}$ for all $a, b \in$ $[0,1]$. Then $(X, M, *)$ is a fuzzy metric space. Define $A=B$ and $T=S: X \rightarrow X$ by $A x=\sin (x /(1+x))$ and $T x=\sin x$ for all $x \in X$. Then we have the following:

(1) $A$ and $S$ satisfy the property (E.A) for the sequence $x_{n}=1 / n, n=1,2, \ldots$,

(2) $A$ and $S$ are weakly compatible,

(3) $A X=[0, \sin (1 / 2)] \subset T X=[0, \sin 1]$, and $A X, T X$ are closed;

(4) (C1) holds. In fact, if $\left\{x_{n}\right\}$ and $\left\{y_{n}\right\}$ in $X$ and $0<\lim _{n \rightarrow \infty} \operatorname{Min}\left(x_{n}, y_{n}, t\right)=L(t)<1$, then there exists a subsequence $\left\{\operatorname{Min}\left(x_{n_{i}}, y_{n_{i}}, t\right)\right\}$ such that $\lim _{i \rightarrow \infty} \operatorname{Min}\left(x_{n_{i}}, y_{n_{i}}, t\right)=L(t)$. Since $\left\{x_{n}\right\}$ and $\left\{y_{n}\right\}$ in $X,\left\{x_{n}\right\}$ and $\left\{y_{n}\right\}$ have convergent subsequence. With out of generality, assume that $\lim _{i \rightarrow \infty} x_{n_{i}}=x_{0}$, and $\lim _{i \rightarrow \infty} y_{n_{i}}=y_{0}$. Then we have that

$$
\begin{aligned}
\lim _{i \rightarrow \infty} M & \left(A x_{n_{i}}, B y_{n_{i}}, t\right) \\
= & \frac{t}{t+\left|\sin \left(x_{0} /\left(1+x_{0}\right)\right)-\sin \left(y_{0} /\left(1+y_{0}\right)\right)\right|}, \\
\lim _{i \rightarrow \infty} \operatorname{Min}\left(x_{n_{i}}, y_{n_{i}}, t\right) & \\
=\min & \left\{\frac{t}{t+\left|\sin x_{0}-\sin y_{0}\right|},\right. \\
& \frac{t}{t+\left|\sin x_{0}-\sin \left(x_{0} /\left(1+x_{0}\right)\right)\right|}, \\
& \frac{t}{t+\left|\sin y_{0}-\sin \left(y_{0} /\left(1+y_{0}\right)\right)\right|}, \\
\frac{t}{t+\left|\sin x_{0}-\sin \left(y_{0} /\left(1+y_{0}\right)\right)\right|}, & t
\end{aligned}
$$

If $0 \leq x_{0}<y_{0}$, then

$$
\begin{aligned}
L(t)=\min \left\{\frac{t}{t+\sin y_{0}-\sin x_{0}},\right. \\
\frac{t}{t+\sin x_{0}-\sin \left(x_{0} /\left(1+x_{0}\right)\right)}, \\
\frac{t}{t+\sin y_{0}-\sin \left(y_{0} /\left(1+y_{0}\right)\right)}, \\
\left.\frac{t+\left|\sin x_{0}-\sin \left(y_{0} /\left(1+y_{0}\right)\right)\right|}{t+\sin y_{0}-\sin \left(x_{0} /\left(1+x_{0}\right)\right)}\right\} .
\end{aligned}
$$


It is clear that

$$
\begin{gathered}
\sin y_{0}-\sin \frac{x_{0}}{1+x_{0}}>\sin y_{0}-\sin x_{0}, \\
\sin y_{0}-\sin \frac{x_{0}}{1+x_{0}}>\sin x_{0}-\sin \frac{x_{0}}{1+x_{0}}, \\
\sin y_{0}-\sin \frac{x_{0}}{1+x_{0}}>\sin y_{0}-\sin \frac{y_{0}}{1+y_{0}} .
\end{gathered}
$$

It follows from

$$
\sin y_{0}+\sin x_{0}>\sin \frac{x_{0}}{1+x_{0}}+\sin \frac{y_{0}}{1+y_{0}}
$$

that

$$
\sin y_{0}-\sin \frac{x_{0}}{1+x_{0}}>\sin \frac{y_{0}}{1+y_{0}}-\sin x_{0}
$$

On the other hand,

$$
\begin{gathered}
\sin y_{0}-\sin \frac{x_{0}}{1+x_{0}}>\sin x_{0}-\sin \frac{x_{0}}{1+x_{0}} \\
>\sin x_{0}-\sin \frac{y_{0}}{1+y_{0}} .
\end{gathered}
$$

Thus, we have that

$$
\sin y_{0}-\sin \frac{x_{0}}{1+x_{0}}>\left|\sin x_{0}-\sin \frac{y_{0}}{1+y_{0}}\right|
$$

It follows from those inequalities that

$$
L(t)=\frac{t}{t+\sin y_{0}-\sin \left(x_{0} /\left(1+x_{0}\right)\right)} .
$$

Similarly, if $0 \leq y_{0}<x_{0}$, then we can get that

$$
L(t)=\frac{t}{t+\sin x_{0}-\sin \left(y_{0} /\left(1+y_{0}\right)\right)},
$$

and if $x_{0}=y_{0}>0$, then we have that

$$
L(t)=\frac{t}{t+\sin x_{0}-\sin \left(x_{0} /\left(1+x_{0}\right)\right)} .
$$

Thus, we have that

$$
L(t)= \begin{cases}\frac{t}{t+\sin y_{0}-\sin \left(x_{0} /\left(1+x_{0}\right)\right)} & \text { if } 0 \leq x_{0}<y_{0} \\ \frac{t}{t+\sin x_{0}-\sin \left(y_{0} /\left(1+y_{0}\right)\right)} & \text { if } 0 \leq y_{0}<x_{0} \\ \frac{t}{t+\sin x_{0}-\sin \left(x_{0} /\left(1+x_{0}\right)\right)} & \text { if } x_{0}=y_{0}>0\end{cases}
$$

It is clear that

$$
\begin{gathered}
\frac{t}{t+\sin \left(y_{0} /\left(1+y_{0}\right)\right)-\sin \left(x_{0} /\left(1+x_{0}\right)\right)} \\
>\frac{t}{t+\sin y_{0}-\sin \left(x_{0} /\left(1+x_{0}\right)\right)} \\
\quad \text { if } 0 \leq x_{0}<y_{0} ; \\
\frac{t \quad \sin \left(y_{0} /\left(1+y_{0}\right)\right)}{t+\sin \left(x_{0} /\left(1+x_{0}\right)\right)-\sin \left(y_{0} /\left(1+y_{0}\right)\right)} \\
\text { if } 0 \leq y_{0}<x_{0} ; \\
1>\frac{t \quad \text { if } x_{0}=y_{0}>0 .}{t+\sin x_{0}-\sin \left(x_{0} /\left(1+x_{0}\right)\right)}
\end{gathered}
$$

This shows that

$$
\begin{aligned}
\varlimsup_{n \rightarrow \infty} & M\left(A x_{n}, B y_{n}, t\right) \\
\geq & \lim _{i \rightarrow \infty} M\left(A x_{n_{i}}, B y_{n_{i}}, t\right)>L(t) \\
& =\lim _{i \rightarrow \infty} \operatorname{Min}\left(x_{n_{i}}, y_{n_{i}}, t\right)=\underline{\lim _{n \rightarrow \infty}} \operatorname{Min}\left(x_{n}, y_{n}, t\right) .
\end{aligned}
$$

Thus, (C1) holds.

(5) By Theorem 9, $A$ and $T$ have common fixed point.

Example 20. Let $X=[0,1], M(x, y, t)=t /(t+|x-y|)$ for every $x, y \in X$ and $t>0$ and $a * b=\min \{a, b\}$ for all $a, b \in$ $[0,1]$. Then $(X, M, *)$ is a fuzzy metric space. Define $A=B$ and $T=S: X \rightarrow X$ by

$$
A x= \begin{cases}\frac{1}{2} & \text { if } x=0 \\ 0 & \text { if } x=1 \\ \frac{x}{2} & \text { if } x \in(0,1)\end{cases}
$$

and $T x=x$ for all $x \in X$. Then we have the following:

(1) $A$ and $S$ satisfy the property (E.A) for the sequence $x_{n}=1 / n, n=1,2, \ldots$;

(2) $A$ and $S$ are weakly compatible;

(3) $A X=[0,1 / 2] \subset T X=[0,1]$, and $A X, T X$ are closed; 
(4) (C1) does not hold. In fact, for $x_{n}=1 / n$ and $y_{n}=0$, $n=1,2, \ldots$, we have that

$$
\begin{aligned}
\operatorname{Min}\left(x_{n}, y_{n}, t\right) & \\
=\min & \left\{M\left(\frac{1}{n}, 0, t\right), M\left(\frac{1}{n}, \frac{1}{2 n}, t\right), M\left(0, \frac{1}{2}, t\right),\right. \\
& \left.M\left(\frac{1}{n}, \frac{1}{2}, t\right), M\left(0, \frac{1}{n}, t\right)\right\} \\
= & \min \left\{\frac{t}{t+(1 / n)}, \frac{t}{t+(1 / 2 n)}, \frac{t}{t+(1 / 2)},\right. \\
& \left.\frac{t}{t+(1 / 2)-(1 / n)}, \frac{t}{t+(1 / n)}\right\} \rightarrow \frac{t}{t+(1 / 2)}
\end{aligned}
$$

$$
\begin{aligned}
\varlimsup_{n \rightarrow \infty} M\left(A x_{n}, B y_{n}, t\right) \\
=\varlimsup_{n \rightarrow \infty} M\left(\frac{1}{2 n}, \frac{1}{2}, t\right) \\
=\varlimsup_{n \rightarrow \infty} \frac{t}{t+(1 / 2)-(1 / 2 n)}=\frac{t}{t+(1 / 2)} .
\end{aligned}
$$

Thus, (C1) does not hold.

(5) $A$ and $T$ have no common fixed point.

Example 19 does not satisfy the $\varphi$-type contractive conditions used in [10-16]. Example 20 shows that if $(C 1)$ does not hold, then $A$ and $T$ may have no common fixed point. Thus, $(C 1)$ is important for the existence of common fixed point.

\section{Acknowledgments}

The authors would like to thank the referees for useful comments and suggestions. This research was supported by the NNSF of China (fund no. 11171286).

\section{References}

[1] L. A. Zadeh, "Fuzzy sets," Information and Computation, vol. 8, pp. 338-353, 1965.

[2] A. George and P. Veeramani, "On some results in fuzzy metric spaces," Fuzzy Sets and Systems, vol. 64, no. 3, pp. 395-399, 1994.

[3] O. Kaleva and S. Seikkala, "On fuzzy metric spaces," Fuzzy Sets and Systems, vol. 12, no. 3, pp. 215-229, 1984.

[4] I. Kramosil and J. Michálek, "Fuzzy metrics and statistical metric spaces," Kybernetika, vol. 11, no. 5, pp. 336-344, 1975.

[5] J. Rodríguez-López and S. Romaguera, "The Hausdorff fuzzy metric on compact sets," Fuzzy Sets and Systems, vol. 147, no. 2, pp. 273-283, 2004.

[6] K. Menger, "Statistical metrics," Proceedings of the National Academy of Sciences of the United States of America, vol. 28, pp. 535-537, 1942.

[7] V. Gregori and S. Romaguera, "Some properties of fuzzy metric spaces," Fuzzy Sets and Systems, vol. 115, no. 3, pp. 485-489, 2000 .
[8] A. George and P. Veeramani, "On some results of analysis for fuzzy metric spaces," Fuzzy Sets and Systems, vol. 90, no. 3, pp. 365-368, 1997.

[9] V. Gregori and S. Romaguera, "On completion of fuzzy metric spaces," Fuzzy Sets and Systems, vol. 130, no. 3, pp. 399-404, 2002.

[10] D. Gopal, M. Imdad, and C. Vetro, "Impact of common property (E.A.) on fixed point theorems in fuzzy metric spaces," Fixed Point Theory and Applications, vol. 2011, Article ID 297360, 14 pages, 2011.

[11] M. Imdad and J. Ali, "Some common fixed point theorems in fuzzy metric spaces," Mathematical Communications, vol. 11, no. 2, pp. 153-163, 2006.

[12] M. Imdad, J. Ali, and M. Hasan, "Common fixed point theorems in fuzzy metric spaces employing common property (E.A.)," Mathematical and Computer Modelling, vol. 55, no. 3-4, pp. 770778, 2012.

[13] D. Miheț, "Fixed point theorems in fuzzy metric spaces using property E.A," Nonlinear Analysis, vol. 73, no. 7, pp. 2184-2188, 2010.

[14] Y. Shen, D. Qiu, and W. Chen, "Fixed point theorems in fuzzy metric spaces," Applied Mathematics Letters, vol. 25, no. 2, pp. 138-141, 2012.

[15] C. Vetro and P. Vetro, "Common fixed points for discontinuous mappings in fuzzy metric spaces," Rendiconti del Circolo Matematico di Palermo, vol. 57, no. 2, pp. 295-303, 2008.

[16] P. Vijayaraju and Z. M. I. Sajath, "Some common fixed point theorems in fuzzy metric spaces," International Journal of Mathematical Analysis, vol. 3, no. 15, pp. 701-710, 2009.

[17] M. Grabiec, "Fixed points in fuzzy metric spaces," Fuzzy Sets and Systems, vol. 27, no. 3, pp. 385-389, 1988.

[18] M. Abbas, I. Altun, and D. Gopal, "Common fixed point theorems for non compatible mappings in fuzzy metric spaces," Bulletin of Mathematical Analysis and Applications, vol. 1, no. 2, pp. 47-56, 2009.

[19] G. Jungck, "Common fixed points for noncontinuous nonself maps on nonmetric spaces," Far East Journal of Mathematical Sciences, vol. 4, no. 2, pp. 199-215, 1996. 


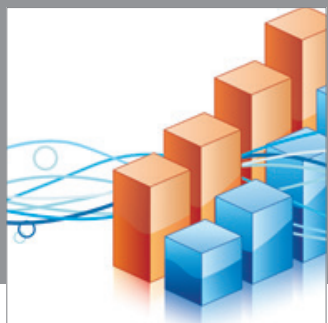

Advances in

Operations Research

mansans

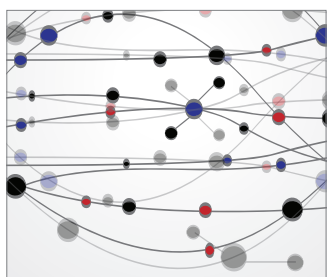

The Scientific World Journal
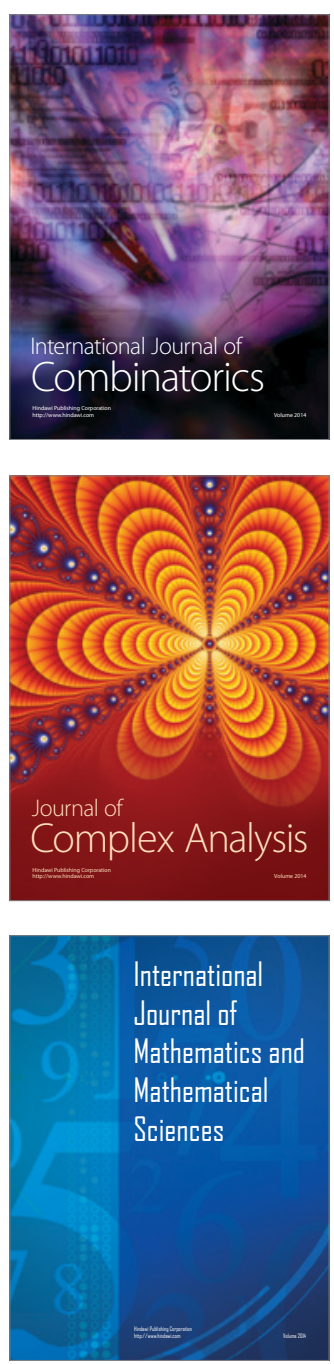
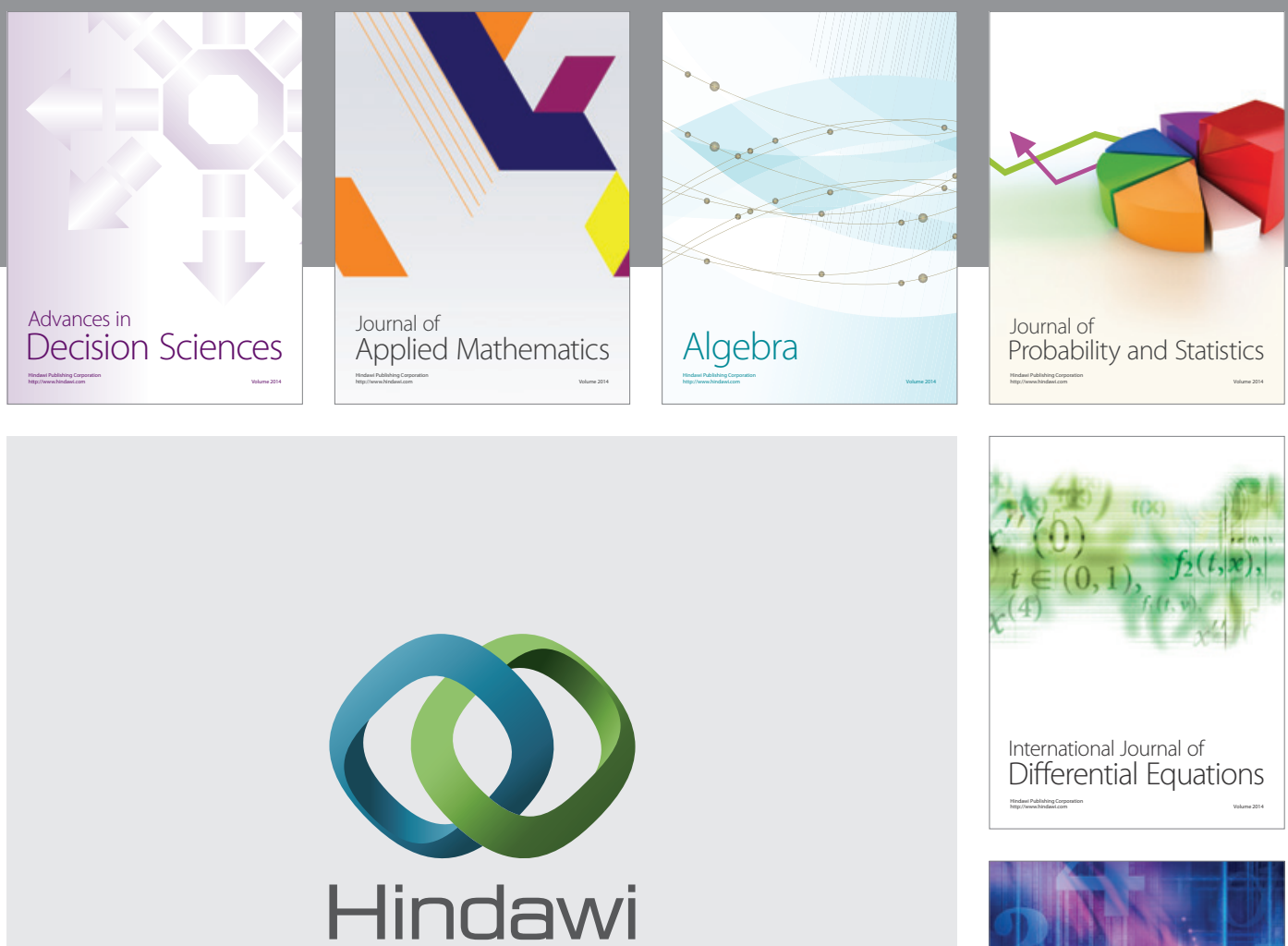

Submit your manuscripts at http://www.hindawi.com
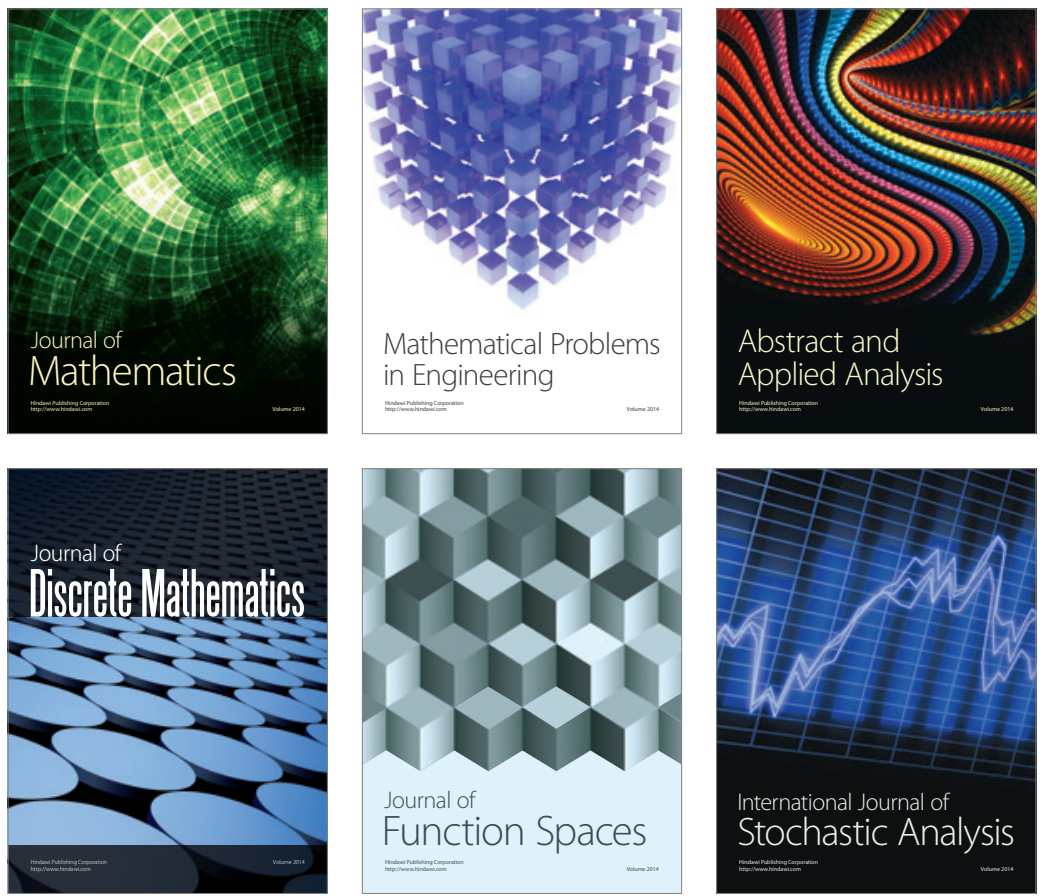

Journal of

Function Spaces

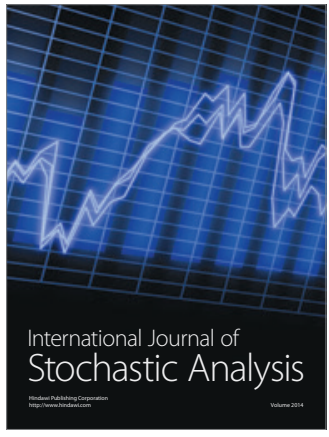

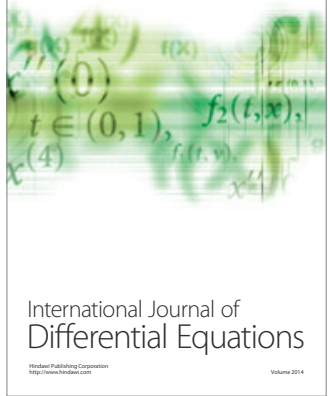
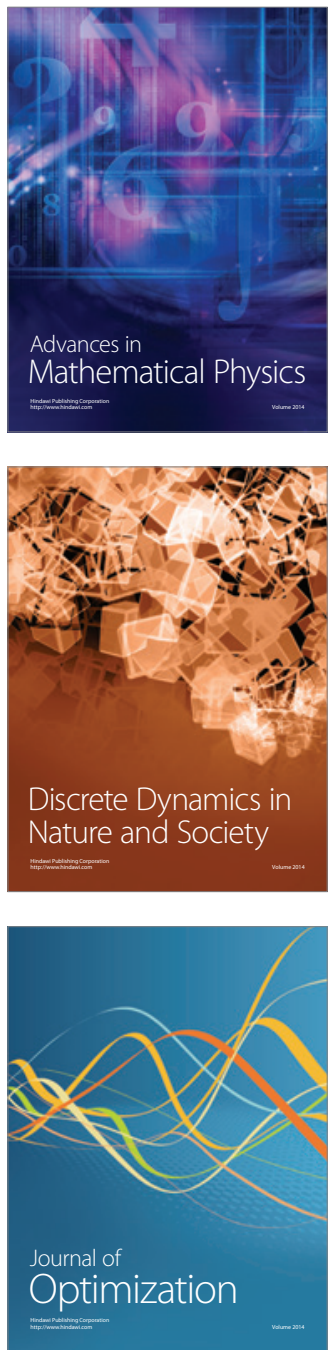